\title{
Determining the Utility of Pathologic Analysis of Ureteral Specimens Obtained from Repair of Vesicoureteral Reflux and Ureterovesical Junction Obstruction in Children: Potential for Physician-Initiated Cost-Reduction ${ }^{*}$
}

\author{
Unwanaobong Nseyo, Mark R. Anderson, John S. Wiener ${ }^{\#}$ \\ Division of Urologic Surgery, Department of Surgery, Duke University Medical Center, Durham, USA \\ Email: \#john.wiener@duke.edu
}

Received October 28, 2013; revised November 20, 2013; accepted November 28, 2013

Copyright (c) 2013 Unwanaobong Nseyo et al. This is an open access article distributed under the Creative Commons Attribution License, which permits unrestricted use, distribution, and reproduction in any medium, provided the original work is properly cited. In accordance of the Creative Commons Attribution License all Copyrights (C) 2013 are reserved for SCIRP and the owner of the intellectual property Unwanaobong Nseyo et al. All Copyright @ 2013 are guarded by law and by SCIRP as a guardian.

\begin{abstract}
Purpose: Partial resection of the distal ureter is commonly performed during reconstructive surgery in pediatric urology; particularly for correction of vesicoureteral reflux (VUR) and ureterovesical junction obstruction (UVJO). Many hospitals require pathologic examination of all excised tissues. We examined the pathologic findings in such specimens to evaluate the utility of this practice. Methods: We reviewed the findings on pathologic examination of distal ureteral segments excised during surgical correction of VUR and UVJO in children by a single surgeon over a 13-year period. Results: One hundred and ninety-one specimens from 126 patients were reviewed. None were found to have any significant pathologic findings that impacted therapy. Conclusion: Routine pathological examination of distal ureteral segments excised during surgical correction of VUR and UVJO is not warranted. This presents potential for cost and resource reduction in care of these patients.
\end{abstract}

Keywords: Vesicoureteral Reflux; Ureterovesical Junction Obstruction; Surgical Reimplantation; Ureteral Pathology

\section{Introduction}

Both vesicoureteral reflux (VUR) and ureterovesical junction obstruction (UVJO) are the result of a congenital malformation of the ureterovesical junction. VUR is a relatively common pediatric urological condition present in up to $1 \%$ to $2 \%$ of all children, while UVJO is a much less common disease. Surgical intervention is warranted for UVJO when renal function is impacted, while, controversies exist regarding diagnosis and management of VUR. Surgical correction remains a mainstay of treatment and can be achieved via an open intravesical or extravesical approach or, more recently, by endoscopic injection of a bulking agent. An analysis of correction of VUR at 40 US children's hospitals encompassing more

\footnotetext{
${ }^{*}$ Conflict of Interest: J.S.W has served on advisory panels for Glaxo Smith Kline and Lilly.

Funding Source: None.

Ethical Approval: This study was approved by our institutional review board.

\#Corresponding author.
}

than 15,000 cases over a five-year period found that $63 \%$ of the repairs used an open surgical approach [1]. With the intravesical approach in VUR repair and with repairs of UVJO, a portion of the distal ureter is usually excised prior to reimplantation.

At our hospital, institutional policy requires that all surgical specimens be subject to histopathologic analysis. The only exempt specimens with respect to urological procedures are foreskin from pediatric or adult circumcision, calculi, and penile implants [2]. A protocol exists by which a physician can request examination of a specimen, but no specimen may be withheld from analysis unless it is previously exempt. According to institutional policy, the excised ureteral specimens from surgical repair of VUR and UVJO are submitted for routine histopathologic processing and analysis.

The goal of our study was to identify and confirm the histopathologic findings associated with ureteral tissue excised during surgical repair of VUR and UVJO. In particular, we were concerned with the presence of any 
pathology with a potential to impact patient management. A primary endpoint of the study was to evaluate the costeffectiveness of this practice and as such a cost analysis for the pathological processing of the specimen was also included.

\section{Materials and Methods}

We conducted a single-center, single-surgeon retrospective/historical case series study of patients who underwent open repair of VUR or UVJO from September 1997 to January 2011. Patient data was identified through electronic medical records. We identified all patients with a CPT code for ureteral reimplantation (50780.00unilateral, 50780.50-bilateral, 50783.00-tapered reimplantation, 50782.00 — duplicated system, 50780.22revision) in that time period. Indications for repair were typically breakthrough UTI and lack of resolution for VUR and progressive hydronephrosis, decreased renal function, or UTI in UVJO. Most cases of VUR were higher grade due to surgeon preference to observe most cases of low grade VUR or perform endoscopic correction (once available in 2001) when intervention was warranted. Intravesical ureteral reimplantation techniques was mostly common the Cohen technique with GlennAnderson and Politano-Leadbetter repairs used in select cases. Patients were subsequently selected with the ICD-9 codes for VUR (593.70) or UVJO (753.22). Patients were excluded who did not have pathology specimens in the case of reimplantation without distal ureter excision (e.g. extravesical approach for ureteral reimplantation). Patients were also excluded who were older than 18 years of age at the time of surgery.

The Institutional Review Board at our institution provided approval for the development and analysis of this dataset.

We obtained pathology reports for the selected patients from the electronic medical record and microfilm. All specimens had undergone both gross and microscopic examination. The specimens were routinely processed in formalin and stained with hematoxylin/eosin. The pathology reports for each specimen were evaluated for the presence of the folllowing terms: "malignancy," "abnormality," "inflammation," "fibrosis," "hyperplasia,” “dysplasia," "benign,” and "normal.” We focused on reports with results other than benign or normal ureteral tissue.

The costs associated with pathology analysis and processing of the ureteral specimens were obtained from the Department of Pathology and hospital billing. The financial information was based upon the actual billed amounts standard for the surgical and pathology components of the hospital visit.

\section{Results}

Our retrospective chart review yielded 165 patients who had undergone open surgical repair of VUR or UVJO. Of these, 126 patients had verifiable pathology specimens from 191 ureters. Nine of the patients had UVJO repair, nine had an ureteroneocystotomy for a ureterocele and the remainder had VUR. Two-thirds of the patient population was female. The median age was 4 years of age, with an age range of 2 months to 18 years. There was an equal distribution of right and left specimens. Sixty-four cases yielded bilateral specimens.

None of the 191 ureteral specimens were found to have any ureteral pathology suggestive of malignancy, carcinoma or dysplasia. The most common ureteral specimen finding was that of benign or normal ureteral tissue. The normal ureteral specimens were designated in many cases by stating that the specimen was a ureter, negative for malignancy or dysplasia. The most common pathologic "abnormality" noted was mild, often chronic, inflammatory changes.

As no additional staining was completed, our results were limited to changes that could be identified microscopically. Of the ureteral specimens with identifiable variations from normal tissue, the histological findings included urothelial hyperplasia, chronic inflammation, and chronic ureteritis. Often in cases of UVJO repair, a segment of dilated ureter was also included in the specimen. In a small number of cases, the ureteral specimen showed evidence of acute hemorrhage and electrocautery. There were several cases in which the urothelium was noted as being completed denuded. In one unique case, a tubular specimen was removed that was thought to be the ureter although anatomically attached to the testicle. Ultimately, the specimen was composed of urothelium and determined to be ureter in an abnormal anatomical configuration.

A cost analysis of the charges associated with the pathology processing was conducted and compared to the overall charges of the hospitalization associated with surgical repair of VUR and UVJO. Current charges for each pathology specimen analysis are \$549: \$189 of which is the hospital charge and $\$ 360$ of which are professional fees from the surgical pathologist. Total charges for open surgical repair of VUR/UVJO followed by a two-day hospital stay were $\$ 24,461$ : $\$ 14,741$ was denoted for the hospital charges and $\$ 9720$ for professional fees for the surgeon and the anesthesiologist.

\section{Discussion}

In our review of histopathology of ureteral specimens excised during the open repair of VUR and UVJO in children, no concerning findings were noted. We believe that this is first study to address the lack of meaningful pathologic findings in ureters excised during routine pediatric reconstructive surgery. The only finding noted were benign inflammatory changes associated with the 
mechanism of disease. Chronic inflammation in a patient with VUR can be secondary to the urinary tract infections often associated with VUR. The backflow of urine in VUR may also be responsible for the hyperplastic changes observed. The ureters in individuals with UVJO are thought to be hypoplastic in nature, resulting in abnormal peristalsis $[3,4]$.

Due to the lack of additional staining, the architecture of the ureteral connective tissue could not be examined in this study. A study of children with grade IV and V VUR without concurrent renal pathology found decreased collagen thickness of the ureteral wall compared to controls [5]. Analysis of smooth muscle fiber architecture in distal ureteral specimens excised after correction of VUR demonstrated smooth muscle atrophy, architectural derangement, and decreased fiber expression [6]. The density and architecture of the smooth muscle fibers of the intravesical ureter appear to correlate with the degree of ureteral dilation [7]. Muscle hypertrophy of the ureter proximal to the obstruction defines the histopathologic findings in UVJO and is thought to develop through a compensatory mechanism. Although the body of literature is minimal, these studies establish the benign morphological changes found in ureters with VUR and UVJO which fail to provide any clinically relevant information. The morphological changes and histological changes found in our specimens in no way impacted further management of patients following surgical repair of VUR or UVJO.

The histopathologic findings in our study have a potential to inform policy change within our institution as there is potential for cost-savings. Currently, ureteral specimens excised during the repair of VUR and UVJO must undergo mandatory gross and microscopic pathologic examination. Utilizing the cost analysis from above, the pathology charges, therefore, represents $2.2 \%$ of the total charges for the procedure, but this proportion would likely rise as hospital charges have trended downward with shorter lengths of stays following open surgery for VUR. Modest savings could, therefore, be realized with our proposed elimination of histopatholgic analysis of excised ureteral tissue in correction of VUR and UVJO. Additionally, hospital resources can be used on more clinically impactful efforts. We believe that it is ethically sound to take this step to reduce financial and health care resources with little to no perceivable detriment to the patient.

Limitations of our study include its retrospective nature. Also we were limited to pathology reports and therefore were not able to verify the findings or potentially stains for connective tissue analysis. Over the period of the study, healthcare costs and delivery have changed; for instance, the length of stay following many surgical procedures in children has decreased. Also, when looking for a very rare event, it is possible that our sample size was insufficient to detect potentially deleterious pathology; however, the benign nature of the congenital disorders examined makes that possibility highly unlikely. Regardless, if elements of the repair are not routine, it is reasonable to submit the excised ureteral tissue for histopathologic analysis.

\section{Conclusion}

Our review of histopathologic analysis of ureters excised during a 13-year experience with the open repair of VUR and UVJO in children found no clinically significant pathology. The uniformly benign histological changes noted had no influence upon clinical management of these patients. Elimination of routine histopathological analysis of ureteral tissue excised during repair of these congenital anomalies could result in cost reduction in the management of these disorders in children.

\section{REFERENCES}

[1] J. C. Routh, C. P. Nelson, D. A. Graham and T. A. Lieu, "Variation in Surgical Management of Vesicoureteral Reflux: Influence of Hospital and Patient Factors," Pediatrics, Vol. 183, 2010, pp. 1568-1572.

[2] “Tissue Medical Devices Exemption List,” Health Systems Policies, Duke University Health System.

[3] M. Murakumo, K. Nonomura, T. Yamashita, T. Ushiki, K. Abe and T. Koyanagi, "Structural Changes of Collagen Components and Diminution of Nerves in Congenital Ureteropelvic Junction Obstruction,” The Journal of Urology, Vol. 157, No. 5, 1997, pp. 1963-1968. http://dx.doi.org/10.1016/S0022-5347(01)64910-3

[4] J. M. Park and D. A. Bloom, "The Pathophysiology of UPJ Obstruction. Current Concepts,” Urologic Clinics of North America, Vol. 25, No. 2, 1998, pp. 161-169. http://dx.doi.org/10.1016/S0094-0143(05)70004-5

[5] M. Yurtcu, G. Nilifer, F. Siddika, C. A. Mustafa and G. Engin, "Investigation of Histopathologic Changes in the Ureter Walls in Vesicoureteral Reflux," Journal of Pediatric Surgery, Vol. 44, No. 4, 2009, pp. 802-805. http://dx.doi.org/10.1016/j.jpedsurg.2008.08.018

[6] J. Oswald, E. Brenner, C. Schwentner, M. Deibl, G. Bartsch, H. Fritsch and C. Radmayr, "The Intravesical Ureter in Children with Vesicoureteral Reflux: A Morphological and Immunohistochemical Characterization,” The Journal of Urology, Vol. 170, No. 6, 2003, pp. 2423-2427. http://dx.doi.org/10.1097/01.ju.0000097146.26432.9a

[7] B. Lee, A. Partin, J. Epstein, D. Qiunlan, J. Goslin and J. Gearhart, "A Quantitative Histological Analysis of the Dilated Ureter of Childhood," The Journal of Urology, Vol. 148, 1991, pp. 1482-1486. 


\section{Abbreviations}

CPT: Current Procedural Terminology

ICD: International Statistical Classification of Diseases and Related Health Problems

VUR: Vesicorureteral Reflux

UVJO: Ureterovesical Junction Obstruction 